\title{
Spatializing the Musicking of an Expressive Urban Imagination: A Trans-Cultural Evaluation of The Early- Modern Rock Music of Bengal.
}

\author{
Shankhadeep Chattopadhyay \\ Research Scholar, Department of English, Banaras Hindu University, Varanasi, 221005, \\ India, shankhamanu@gmail.com, ORCID id - ooo-0oo3-4678-3031.
}

\begin{abstract}
The technological reproduction of the imaginary has always reflected a polarization in urban consciousness, considering the city as the urban 'body' - which retains a space for contemporary imaginations. Rock music of the American 6os radiates exactly such urban consciousness by constantly experimenting with lyrics, sounds, images and celebrations, which continually harmonize with the changing industrial and technocratic city structures. This paper explores a progressive cultural synthesis between the American and the earlymodern rock music of Bengal. The city of Kolkata in West Bengal has always been repleted with a vibrant 'representational space' with a high rate of western-music consumption since the late 1970s, thus reflecting western urban ethos into the Indian urban imagination through modern Bengali rock music. Lefebvre (1974) suggests that the potential for genuine social change is possible only through the city as practised rather than the city as planned; on this note, this paper analyzes how the Indian urban imagination negotiated with the everyday urban experience of distant musical and cultural behaviours through 'musicking' by producing a musically reflective space where thought, feelings and different moods are crafted and performed. Further, how, in the age of technical reproduction, rock music produces a 'counter-space' by projecting urban ethos, which acts as an exegetic tool for the symptomatic reading of any expressive culture, and makes the city claim its spatial identity.
\end{abstract}

Keywords: representational space, counterculture, rock music, urban ethos, musicking.

\section{Introduction}

Now I will do nothing but listen,

To accrue what I hear into this song, to let sounds contribute toward it.

I hear bravuras of birds, bustle of growing wheat, gossip of flames, clack of

sticks cooking my meals,

I hear the sound I love, the sound of the human voice,

I hear all sounds running together, combined, fused or following,

Sounds of the city and sounds out of the city, sounds of the day and night...

- Walt Whitman. Song of Myself (Section 26, Line 1-7).

(c) AesthetixMS 2021. This Open Access article is published under a Creative Commons Attribution Non-Commercial 4.0 International License (http://creativecommons.org/licenses/by-nc/4.o/), which permits non-commercial re-use, distribution, and reproduction in any medium, provided the original work is properly cited. For citation use the DOI. For commercial re-use, please contact editor@rupkatha.com. 
The mystery of hearing sounds is always an isolated figment of some sort of action taking place in the abstract plane, creating totality and forming language for the production of aesthetics by which we articulate the relationships of our lives to all that of socio-political and economic circumstances. In the widest sense this action is after all political, because it helps fertilize a space where a set of relationships interplay and makes a point of departure for the listening subject from the everyday lives, solely for the purpose of listening. Small (1998) found that this act of listening takes a significant part in 'musicking' - the extra 'ing' is signifying music as a 'verb', a process and not as a noun or a 'thing'. Small claims that there's no such thing called "music", it's not a "thing" at all, but "an activity, something people do" (p. 11). This activity takes place in the symbolic realm between the performers and listeners-lyrics, gesture, reception and various other tools - related to the total musical experience. The symbolic negotiations achieve a degree of flexibility and help us, as Small (1998) suggests,

[In] knowing our world - not that given physical world, divorced from the human experience, that modern science claims to know but the experimental world of relationships in all its complexity- and in knowing it, we learn how to live well in it. (p. 5o)

Hence, musicking is an important aspect for the understanding of music's symbolic relationship with places, especially the city, as it is the lynchpin of music production, distribution and consumption, vis-à-vis it plays a pivotal role in characterizing spaces. Instead of considering music as a 'thing', Small proposes it to be a trap of "reification" or "thing making", which has dominated the western thought at least from the time of Plato (p. 61). The places of 'musicking' in the 6os and 7os America were highly spatialized as they represented the value of the contemporary industrial world and made the concept of sociability inclined to the musical experience of the age. Be it Dylan's outrageous protest songs or Jim Morrison's euphoric urban Dionysia, all signify different places of 'musicking' and represent various degrees of rejection to the Apollonian order of existence. 6os popular imagination was arguably influenced and shaped by music, which acted as a socializing agent and retained its specific agency, as Krims (2007) would suggest, by "relying crucially on, and sometimes acting on, its specificity as sound that it organized and continually reproduced in a developing social context" (p. 20).

The wave of countercultural song tradition in America splashed upon the Indian shore at a time when the young nation was struggling to cope with overwhelming sociopolitical problems and witnessing extraordinary convulsions. While the villages were projected as representations of real India at that point of time, the urban imagination had its own emphatic concerns to express as well. Large and expansive cities like Bombay, Kolkata, Madras and Delhi along with Shimla and Bangalore were the epicenters of western music consumption and throughout the swinging decade, parallel to that of American and British counterculture, these places remained vastly productive and offered a significant series of Rock, Jazz, Psychedelic and Blues music. By the 6os, with the euphoria of independence slowly subsiding and the Nehruvian vision of nation-building and 
communal harmony overshadowing the contemporary socio-economic challenges, rock music, according to Bhatia (2014),

became a metaphor for rebellion and angst among a new generation in those heady times...No sooner had a trend taken off in America or Britain than it was picked up in Bombay, Madras and Calcutta. Pop, protest music, rock, hard rock, - they played them all. (Introduction)

Its repository may apparently be insignificant to that of American 6os, but the distant cultural negotiations significantly added up to the construction of the urban Indian youth, who were growing up in the restrictive socio-economic condition and vehemently sneered at bourgeois interests. They were the midnight's children of socialist India and some of them were much ahead of their times.

This article is not based on the study of musicological aspects of the songs produced by the Indian urban imagination, but rather emphasizes on the socio-historical shift in the construction of the urban sounds and of the urban imaginaries in 7os Kolkata. The article specifically critiques the spatial contours and the trans-cultural significance of the inception of Rock music in Kolkata. It also explains how 'musicking' by an expressive urban imagination has broken the deadlock of a violent silence in alternative Bengali music by the formation of new urban ethos.

Edward Soja has observed that Lefebvre's theory of trialectics is an indirect critique to historicism, the reason for which delves deep into the assertion of spatiality. He points out two outcomes from the assertion. One is "historicality" and the other is "sociality" (p. 72). Both significantly contribute to the interpretations and knowledge of human life, as Soja (1996) explains, "while simultaneously maintaining the rich insights they provide for understanding of the production of lived space" (p. 72). Lefebvrian understanding of history is non-linear and a refusal against the teleological progression, which echoes the Nietzschean conception of time-cycles and change. Lefebvre (1974) has talked about the significance of "moment" or instant, which is an immanent critique of the everyday life and has the true potential to alter the existing social order (p. 399). This article will also theorize the spatial inclination of music and city on the backdrop of Lefebvre's theory of spatiality in order to identify the 'musicking place' as "representational space" (p. 33). This observation will reflect the struggle between the production and reproduction of "counterspace" with that of the state dominated production of space through 'musicking' in the urban sphere (p. 381). For the trans-cultural evaluation, I will be focusing on the first ever Bengali rock band, Moheener Ghoraguliii [Moheen's Horses] ${ }^{\text {ii }}$ along with few iconic rock songs from the American 6os to critique the symbolic exchange of the urban imaginaries and ethos.

\section{Moheener Ghoraguli: The Sound of Changing Urban Ethos and Imaginaries}

Alternative cultural voices have historically been silenced by the mainstream, mediated through the relations of power, but expression of such marginalized voices have always illuminated the global alliance with music being their platform in the struggle for local 
spaces. 'Musicking' inside any counterculture significantly adds up to the act of resistance against the homogenizing force of culture industry and asserts new styles. Moheener Ghoraguli's strategies of musical poetics configured a complex subjective unity and projected a new imagination which involves the lyrical and musical characterization of cities. This new urban imagination found a sense of 'place' which negotiated with a range of social activities, and obviously was transmitted from the alternative social voices. Their music represents the city under the changing regime of capital accumulation through expanding spatial practices of new urban working populations. Moheener Ghoraguli's song Shongbigno Pakhikul [Flock of birds on tenterhooks], which came out in 1977 radically altered the perception of 'the urban' by foregrounding everyday places from a completely different point of view that has never been envisioned by Bengali popular imagination before:

No one's across the runway but emptiness

Storm-clouds brooding in the sky

Indolence of the melancholic radar

A few little happy birds on tenterhooks!

[“রানওয়ে জুড়ে পড়ে আছে শুধু কেউ নেই শূন্যতা

আকাশে তখন থমকিয়ে আছে মেঘ

বেদনা বিধুর রাডারের অলসতা

কিঞ্চিত সুখী পাখিদের সংবেদ!’]

One can find a range of construction of the urban images in this representation in a given time span. Krims (2007) suggests these types of representations which come under a given time as "urban ethos" (p. 19). He says that,

Changes in the very outer boundaries of the urban ethos suggest the inseparability of representation and the spatial contours of cities themselves, because they point to 'conditions of possibility' for expressive culture in changing regimes of capital accumulation. (p. 6o)

The possible modalities of representation of the urban image produces distinctions among the city subjects that exist in and move through urban space and obviously with certain extent of freedom (Krims, 2007, p. 58). Here, an abstract condition of a state of void is attempting to impersonalize the runway, because emptiness has not been seen as a state of mind (which is mostly the case in early-modern Bengali music), but becomes a visible object. The very opening of the song portrays a contrast between the condition of weather and of the utilitarian purpose of a runway. The radars are ruffled and reluctant to be unfurled above the sky since it is cloudy, and a group of birds on tenterhooks is a metaphor for the thinking poetic selves of the generation which doesn't completely negate the esperanza of social change, and is therefore concerned about the future outcomes. Such representation severely contributes to the changing urban ethos, as the narrator says: 
The wandering minstrel has seen this image,

And the heart has realized vacant capricious clouds

The wandering minstrel does not hear this image

And so, emerges his affection, his woe

And the cloud in the sky awaits the impending storm.

[চারণ দেখেছে এই ছবিখানি, তাই

হৃদয় জেনেছে শূন্যতা, উড়ুুেঘ

চারণ শোনেনা এই ছবিখানি, তাই

বড়ো মায়া লাগে, বড়ো তার উদ্বেগ

আকাশে তখন ঝড় এসে যাবে বলে থমকিয়ে আছে মেঘ]

The expressive urban concern, which paints the city as utterly a void, echoing a hollowness from within-limited by its experimentation with new artistic forms, cocooned inside the Victorian morality and lacking the global expansion-has represented everyday life and its situations which, Lefebvre (1974) would suggest in another context, are "co-extensive with and utterly transformed by a theatricality as sophisticated as it is unsought, a sort of involuntary mise en scène" (p. 74). This song, especially in the last two lines, acts as a constant reminder of something very ominous, as if the upcoming metaphorical storm would gush through the veins of the stubborn society, knocking all cohesiveness and rules of consistency down. Hence, the narrator delves deep inside the lived experience of the city, pointing out the redolent image of a troubadour who can't accept everything of his society and is utterly anxious, envisaging a series of social problems, like the doom of creative minds inside an orthodox culture and the urgent need of its regeneration. This new urban phenomenon, mobilizing around new spatial consciousness, put forward an open-ended politics of coalition, which severely addressed multiple oppressions of inequality-state, class and on. Creation of the new ethos therefore provides the representational space the quality they demand, of what Lefebvre (1974) explained:

[...] it embraces loci of passion of action and of lived situations and thus immediately implies time [...] it is essentially qualitative, fluid and dynamic [...] representational space produces only the imaginary or symbolic works, they are often unique; sometimes they set in train 'aesthetic' trends and, after a time, having provoked a series of manifestations and incursions into the imaginary, run out of steam. (p. 42)

Another much celebrated song Hay Bhalobasi [Oh! I do love] released in 1977, blatantly depicts the passion for the love of art and alternative music. The very opening of the song with an orchestral soundscape presented by saxophone, violin, guitar and drums, confronts the negation of traditional sound patterns and enables us to access the realm of urban bohemia that harks back to nostalgia and is simultaneously determined to make a symbolic use of music by experimenting with new forms of urban imagination. The song 
writing strategically creates a contrast while expressing the unrestricted passion for a series of romantic non-urban images, the lyrics follow:

Love running amidst the moonlit catkin fields

Love walking on the shadowy rustic roads

Crimsoned by the waning Sun far across the hill

Paddy fields allure me to the green horizon

And yet I wonder why nothing feels good

Seems my mind lounges amidst lackadaisical roads

Keep thinking where the melancholia still stays concealed.

$$
\begin{aligned}
& \text { [ভালবাসি জ্যোৎস্নায় কাশবনে ছুটতে } \\
& \text { ছায়াঘেরা মেঠো পথে ভাল লাগে হাঁটতে } \\
& \text { দুর পাহাড়ের গায়ে গোধূলির আলো নেখে } \\
& \text { কাছে ডাকে ধানক্ষেত সবুজ দিগন্তে } \\
& \text { তবুও কিছুই যেন ভালো যে লাগে না কেন } \\
& \text { উদাসী পথের মারে মন পড়ে থাকে যেন } \\
& \text { কোথায় রয়েছে ভাবি লুকিয়ে বিষাদ তবুও] }
\end{aligned}
$$

All these non-urban cravings are very strategically imagined to represent the essence of Bengali aesthetic and romantic desires, as the imagined wanderer who loves to traverse through the poetic self, is not necessarily an urban persona, but a representative of collective romantic imaginaries of the Bengalis. But the narrator is vehemently upset at the same time amidst all the tranquil setting, and his vision is arrested by the metaphorical road for the flow of life when he sees the working-class people.

When I see them working at the ports or villages,

Reap and sweat only at the stretch of the field.

[যখন দেখি ওরা কাজ করে গ্রানে বন্দরে

শুধুই ফসল ফলায় ঘাম ঝারায় মাঠে প্রান্তরে]

This sudden shift from an array of romantic images to the crude realities of working life signifies some notions of mobility and flexibility inside the imaginary identification of the 'self'. This 'self' is expressing its unabashed longing for the escape in rustic planes, from where it yearns for tranquility but somehow continuously denying the approach for some everyday urban issues, as because it is from where the 'self' belongs and can't escape the responsibility. This continuous shift is tendentiously meant to emphasize on the need of flexibility within the Bengali middle-class imaginations for the reception of western and eastern music altogether. The final stanza directly points out these interests - 
Love Picasso, Bunuel, Dante

Love listening to The Beatles, Dylan and Beethoven

After some Ravisankar and Ali Akbar

Like to come homewards on a misty dawn.

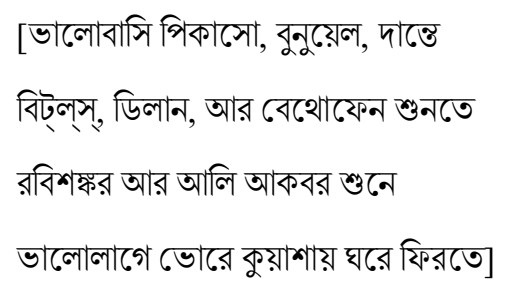

Finally, the song declares its loci of passions, where the 'self' or urban bohemia thrives on nostalgia for distant places, personalities and dreams, attempting to make the immediate place an 'elsewhere' and simply transporting itself away from everyday life. These imaginary movements between objects, people and their desires across places have always been characterized by a spatial dimension of human activity. This was obviously the first attempt to make modern Bengali music global by reflecting the ethos of a distant culture in terms of values and problems, as if replying to the clarion call of Dylan;

Come writers and critics

Who prophesize with your pen

And keep your eyes wide

The chance won't come again

And don't speak too soon

For the wheel's still in spin

And there's no tellin' who

That it's namin'

For the loser now

Will be later to win

For the times they are a-changin'. ['Times They Are A Changin", 1964]

Moheener Ghoraguli felt an extreme immediacy to capture everyday identity in relation with urban places and community, just like the narrator in Simon and Garfunkel's song 'El Condor Pasa', who wishes to become a forest rather than a street, but is somewhere confined and fails in the process of becoming and hence keeps on longing-

I'd rather be a forest than a street

Yes I would, if I could, I surely would. ['El Condor Pasa' (If I Could), 1970] 
This new urban bohemia also tends to be in the rustic plane but cannot help evoking the authenticity inside the lived space. Their 'musicking' creates an "imaginary identification" which establishes the relationship between consumer and performer, where the perceived value of the song-its "emotional conversations"-becomes its exchange value (Connell and Gibson, 2002, p. 28). Here the personal experiences, both real and imaginary, are imbued with emotion and embodied in a narrative form, thus creating an "ideology of authenticity" (Connell and Gibson, 2002, p. 71).

The essential urban landscape of American rock music on the other hand offers trivial and ephemeral visuals which are as diverse as the lyrics, whether it is Jim Morrison's L.A Woman (1971), Bruce Springsteen's Greetings from Asbury Park (1973), or Dylan's Blonde on Blonde (1966), all of which have their source in the clandestine or underground side of social life and stretch across images and symbols that they contain. Lefebvre (1974) suggests this type of place as "representational space" (p. 33), claiming it to be inhabited by the artists, writers and philosophers and also "ethnologists, anthropologists, psychoanalysts [as] students of such representational spaces" (p. 41). This space is passively experienced in the society and can be changed or appropriated by imagination. That means the contemporary urban imaginaries have the true potential to visualize the deep-rooted social issues and overlay physical spaces as they are often marked by a crude history of disenfranchisement and draw heavily on the symbolic relationship of the imaginaries. Hence, they display a set of values which demands personal expressiveness and experimentation that ultimately gives vent with striking clarity towards a conflicting nature of cultural regeneration through the construction of new urban imaginaries.

\section{Symbolic Manifestation of Urban Experiences: The Appropriation of Counter- Space}

The division of labour in the technologically advanced West had produced complex and fragmented social systems as well as alienation. Despite that, some cities shared a privileged history of creativity by remaining vibrantly resistant against the fragmentary nature of this urban alienation. These cities suggested a true global nature in their own as they offered bohemian enclaves for people across nation, race, class, gender and society which led to the bloom of 'representational spaces' for the singers, songwriters, poets and other visionaries. These types of urban spaces always leave a trace in human history and they are, as Soja (1996) explains, "filled with politics and ideology with the real and imagined interwind [...] the 'dominated spaces' [...] the chosen spaces for struggle, liberation, emancipation" (p. 68). Since music and space are dialectically related, they contribute in the expression and shaping of each other, thus creating an awareness against the bourgeois deformation in the society and promoting a "counter-space" (Lefebvre, 1974, p. 381), which runs against the established strategies of power as it embodies, "counterplans and counter-projects designed to thwart strategies, plans and programs imposed from above" (p. 383). Such spaces, produced in the urban sphere, target the hegemonic state, which is the primary organization of space, and involve into an act of struggle through the revolutionary process of musicking, poetry, drama and all other sorts of experimental artistic forms. We find a series of songs from Dylan's lyrical oeuvre like 'Blowin' in the 
Wind', 'Times They Are a Changin', 'Desolation Row', 'Masters of War' and many others which directly address the 'state', and where the counter-plans and projects are razor sharp, expressing an empirical observation on the appropriation of power relations in urban spaces. The process of appropriation takes place symbolically through the construction of urban imaginaries that has already been analyzed in the context of Bengali urban imaginations. Countercultural populations who are the inhabitants of lived space have historically attempted to call for a reform against the state dominated production of space which is homogenizing by nature and therefore fails to transgress the material cohesiveness.

The principal action of Moheener Ghoraguli's later years-even after their separation as a band till their re-emergence in 1995-was dedicated to building touchstones (something remarkable like a saga of liberation) where something protean functioned at the very base of their 'musicking' which captures the symbolic experience of the age. Moheener Ghoraguli's iconic song-Prithibita Naki Choto Hote Hote [As the World Keeps Shrinking Down] which came out in 1995, is perhaps the band's most celebrated anthems which made a wider claim to the new urban imaginations. Unlike the folk and country pattern of the earlier songs, this song emphasizes spontaneity and improvisation in the musical build-up and refuses to move towards traditional celebration or lamentation over any urban landscape. Rather, the song poignantly evokes serious concerns regarding the dark side of technology and how it leads to alienation. The first stanza follows -

As the world keeps shrinking down

To fit in the hands of satellites and cables

Trapped inside an idiot-box in the drawing-room

Ah...ha...,

Sitting at home along with the whole world

Connections clutched in hand today

Swiftly fades away the boundaries of time and distance

Ah...ha...,

Have you ever thought of it?

Faraway as are the stars

Far, far away than that

You and me gradually are drifting apart.

[পৃথিবীটা নাকি ছোট হতে হতে

স্যাটেলাইট আর কেবলের হাতে

ড্রয়িংরুনে রাখা বোকা বাক্সতে বন্দী 


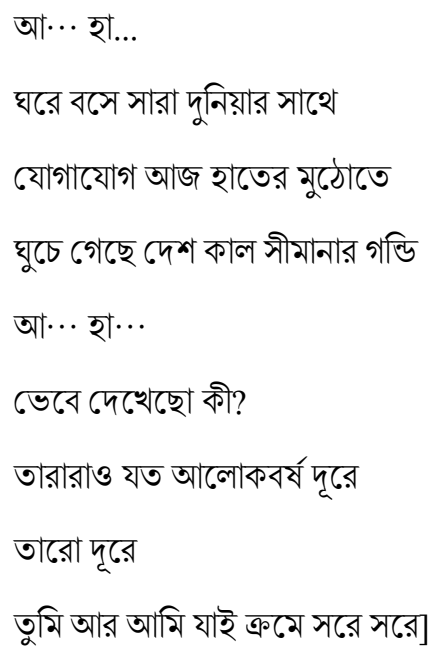

The same narrator who yearns for mobility is concerned at the same time about its aspects here. The singing subject is ridiculing and simultaneously asking question to the alienated selves of the urban Bengali middle class. This provokes questions about the changing notions of human connectivity, both digital and physical. The idiosyncratic symbolic language of the song expresses anxiety of the physical selves, which are drifting apart as technology thrives in the everyday reality with an ominous indication of polarization inside the lived 'social' space of the city.

The process of making this concern understood through the song relies on certain layers of symbolic behaviours, which is "a cerebral process of 'understanding' the world and a set of 'feeling' and 'action' as well as 'knowing" (Martin, 1979), where every day experiences are demonstrated through the new urban imaginaries like, 'the tenant', 'T.V', 'illicit dreams', 'alienation' and others as products of the urban phenomenon which significantly contribute to the characterizing of spaces inside the city. The conscious narrator's vision of the cityscape has changed as it is represented as a place of mischievous desires and traps, much like Jim Morrison's chanting of an urban Dionysia which declared an antediluvian reaction against the American society which was debilitated by capitalism, orthodoxically sustaining hierarchies and feeling smug with achievements at that point of time. Morrison experienced the city scenes majorly over Los Angeles, because it's the city where he spent most of his musical career, which shaped the most fitting epitaph for his band-his sixth studio album for The Doors, L.A Woman (which came out in 1971). The seven-minute-long title track of the album is simply a mash note on the city of Los Angeles, and it goes like:

Well, I just got into town about an hour ago

Took a look around, see which way the wind blow

Where the little girls in their Hollywood bungalows Are you a lucky little lady in The City of Lights?

Or just another lost angel? The

City of Night, City of Night 
... Drive through your suburbs Into your blues, into your blues yeah Into your blues, / into your blues!

I see your hair is burning

Hills are filled with fire

If they say I never loved you

You know they are a liar

Driving down your freeways

Midnight alleys roam

Cops in cars, the topless bars

Never saw a woman so alone

So alone, so alone

Motel money murder-madness

Let's change the mood from glad to sadness. [L.A Woman, 1971]

The urban experiences are obviously much vivid here and expressing several other concerns of urban life, from erotic desires to drugs, brawling, fleeting commitments and pride. The song not just signals for a sordid American apocalypse, but delivers an inciting incantation of Dionysian refusal, a desire to use violence and madness to transcend the social stagnation and fear of alienation, as if any engagement with the very thought of this motif could bring a change in the urban spectacle. All the characters in this song, like the city, the woman and her desired man, are inextricably fabricated to each other's psyche. That is actually the process of gradual connection of the city subjects, who were basically a bunch of disintegrated people, who were existing without any motif of association. Certainly, the horrific portrayal of transformation of a 'city of light' to a 'city of the night' does not only embed in personal connotation but violently seeks for a spiritual resurrection of the city's consciousness.

The representational spaces thus create a locus of tension by envisioning symbolic counter-plans and ideas through 'musicking' in a particular urban space which might shift the control from hegemonic power to the "inhabitants" and "users" of such spaces (p. 39). In this way 'musicking' inside the city can produce or reproduce counter-spaces, the state of which symbolizes a complete uncertainty and reverberates the anxiety through all the other social spaces. The symbolic manifestation of the urban imaginaries over such spaces thus acts as a medium of communication that forms, as Lefebvre (1974) suggested in another context, a "unitary code or the common language of the city" (p. 73). This "unity", he suggested "goes deeper and in as sense higher...it combines the city's reality with its ideality, embracing the practical, the symbolic and the imaginary", hence, severely contributing to the expression of the urban imagination (p. 73-74).

\section{Conclusion}


'Musicking' in all its entirety creates an emphatic understanding in the perpetual comprehension of collective urban imaginaries of any counterculture where images, strategies and existing spatial structures can be studied in the context in which it has arisen. Alternative music across the globe is somehow shaped by international influences, technologies and global values. They interactively and retroactively intervene in the production and reproduction of the spatial contours of society. 'Musicking' places reflect a strategic deployment of relationship between performers and audience, who revalidate their identities by creating symbolic attachments with that place. As the urban population, with all its pros and cons, starts expanding in any city, social experience and reality-both in their unique process of becoming-reflect their projection through new forms of urban images and 'codes'. The formation of counter-space inside 'musicking' places at least temporarily suspends the division between 'private' and the 'public' by unlocking the doors of societal perceptions and does not escape from reality but interrogatively explores the very social organizations. Countercultural song tradition in America has vividly reflected such 'musicking' places throughout its timeline by remaining a major site of encounter inside the repressed zones of the urban body, i.e., the lived city space. Similarly, connected by their unified motif of disaffiliation towards the mainstream culture, Moheener Ghoraguli resurfaced in the modern Bengali music in late 9os by releasing two consecutive albums i.e., Jhora Somoyer Gan [Songs of Withered Time] and Maya [Illusion], in 1996 and 1997 respectively. These albums firmly critique the paramountcy of post-industrial city spaces and more urban issues like unemployment and techno-elitism. Along with Moheener Ghoraguli many other rock bands like Cactus, Chandrabindu and Fossils with their introspective lyrics, distorting guitar effects and heavy sound patterns offered new epistemological groundings for identification of the new Bengali urban youth. As the new millennia wakes up to the expectation and commitment towards more definitive rock and alternative music scene in Bengal, these new bands, like in the constant process of cultural regeneration refill and reproduce the representational space with new ideas, strategies and urban imaginations. Fossils, being first ever rock band to invoke the hymn of sexualliberation and the everyday social concerns regarding sexuality, has been able to produce spontaneous stage performances, inciting their audience to set themselves in the position of a voyeur to experience the sensual and subliminal communion of the artist with a libidinous ecstasy by using the physical apparatus. Their 2004 song, Bicycle Chor [Bicycle Thief] provides a languid and carefree expression of sexuality and politicizes the notion of eroticism for the very first time in the history of Bengali rock music. This revolutionary act of new musicking styles convey their audience the thrill of the unknown, a kind of curiosity, a conglomeration of repugnance, passion and zeal, which significantly contribute to a postindustrial urban imagination. The apparatus of sexual liberation that was hitherto repressed by the moral standards of Bengali middle class, became a transporter of hedonistic ideology that indicated the dawn of a new era. Along with Fossils, contemporary bands like Krosswinds, Chandrabindu, Cactus and Bhoomi all contributed distinctly in the production of psychedelic, progressive and country rock songs and most of them are vibrant still and musicking around the city of joy. 
This article has explored the interconnected urban motifs and ethos which are in constant play inside the imaginary and symbolic realm of a city and also showed how they contribute to the appropriation of 'lived space' thorough 'musicking'. Also, I've attempted to capture the socio-historical shift in the construction of the 'urban' inside the middleclass Bengali imagination through the songs of Moheener Ghoraguli and tried to evaluate them trans-culturally by focusing on the 6os American rock music. This paper provides a spatial approach to the process of 'musicking' which attempts to see through the deeper recesses of urban psyche and incorporates the collective action of social subjects with that of social space, the very arena where, according to Lefebvre (1974), these subjects "develop, give expressions to themselves, and encounter prohibitions; then they perish and that same space contains their grave" (p. 33-34).

\section{Notes}

i. The name of the Bengali rock band Moheener Ghoraguli and others has been italicized by the author for the sole reason of containing the unique Bengali essence while pronouncing the names.

ii. All the Bengali songs and band names in this article have been translated by the author himself.

\section{References}

Banerjee, J. (Ed.). (1988). The Music of Bengal: Essays in Contemporary Perspective. Indian Musicological Society.

Bhatia, S. (2014). India Psychedelic: The Story of A Rocking Generation [kindle edition]. HarperCollins Publisher India. https://www.amazon.in/India-Psychedelic-Story-RockingGeneration-ebook/dp/BooPFWH4SY/ref=tmm_kin_swatch_o?_encoding= UTF8\& qid $=1611334491 \& s r=1-5$

Carlevale, J. (2005). Dionysus Now: Dionysian Myth-History in the Sixties. Arion: A Journal of Humanities and the Classics, 13(2), 77-116. http://www.jstor.org/stable/29737263.

Cohen, S. (2012). Bubbles, Tracks, Borders and Lines: Mapping Music and Urban Landscape. Journal of the Royal Musical Association, 137(1), 135-170. http://www.jstor.org/stable/23321879

Connell, J., \& Gibson, C. (2003). Sound Tracks: Popular Music, Identity, and Place. Psychology Press.

Davis, S. (2011). Jim Morrison: Life, Death, Legend. Random House.

Doors, T. (1971). L.A Woman. On Elektra Records.

Dylan, B. (1964). The Times They Are a- Changin'. On The Times They Are a-Changin' Columbia Records.

---. (1966). Stuck Inside of Mobile with the Memphis Blues Again. On Blonde on Blonde. Columbia Records. 
Ghoraguli, M. (1977). Hay Bhalobasi. On Shangbigno Pakhikul O Kolkata Bishayak. Gathani Records.

---. (1977). Shangbigno Pakhikul. On Shangbigno Pakhikul O Kolkata Bishayak. Gathani Records. Ghoraguli, M. (1995). Prithibita Naki Choto Hote Hote. On Abaar Bachhor Kuri Pore. Asha Audio. Kloosterman, Robert. (2005). Come Together: An Introduction to Music and the City. Built Environment, 31(3). 181-191. http://www.jstor.org/stable/23289438

Krims, A. (2012). Music and Urban Geography. Routledge.

Lefebvre, H. (1992). The Production of Space. Wiley-Blackwell.

Marcus, G. (2013). The Doors: A Lifetime of Listening to Five Mean Years. Hachette UK.

Martin, B. (1979). The Sacralization of Disorder: Symbolism in Rock Music. Sociological Analysis, 40(2), 87-124. doi:10.2307/3709782

Nietzsche, F. (2008). The Birth of Tragedy. Oxford University Press.

Redding, O. (1968). (Sittin' On) The Dock of the Bay. On The Dock of Bay. Stax Records.

Simon, P., \& Garfunkel, A. (1970). El Condor Pasa (If I Could). On Bridge Over Troubled Water. Columbia Records.

Small, C. (2011). Musicking: The Meanings of Performing and Listening. Wesleyan University Press.

Soja, E. (1996). Thirdspace: Journeys to Los Angeles and Other Real-and-Imagined Places. WileyBlackwell.

Springsteen, B. (1973). Greetings from Asbury Park, N.J. On Growin' Up, Columbia Records.

Whitman, W. (2012). Song of Myself. Courier Corporation. 